Bull. Austral. Math. Soc.

VOL. 72 (2005) [87-107]

\title{
ON THE KY FAN INEQUALITY AND RELATED INEQUALITIES II.
}

\author{
EdWARd NEUMan AND JózSEF SÁNDOR
}

Ky Fan type inequalities for means of two or more variables are obtained. Refinements and improvements of known inequalities are derived. Applications to symmetric elliptic integrals of the first and second kind are also included.

\section{INTRODUCTION AND NOTATION}

This paper deals with the Ky Fan type inequalities for means of two and more variables and is a continuation of our earlier work [15].

Let $X=\left(x_{1}, \ldots, x_{n}\right)$ be an $n$-tuple of positive numbers. In what follows the symbols $A_{n}, G_{n}$, and $H_{n}$ will stand for the unweighted arithmetic, geometric, and harmonic means of $X$, respectively, that is,

$$
A_{n}=\frac{1}{n} \sum_{i=1}^{n} x_{i}, \quad G_{n}=\left(\prod_{i=1}^{n} x_{i}\right)^{1 / n}, \quad H_{n}=\frac{n}{\sum_{i=1}^{n}\left(1 / x_{i}\right)} .
$$

We shall always assume that $x_{i} \leqslant \dot{1 / 2}$ for $i=1,2, \ldots, n$. Also, let $X^{\prime}=1-X$ $=\left(1-x_{1}, \ldots, 1-x_{n}\right)$. Throughout the sequel the unweighted arithmetic, geometric, and harmonic means of $X^{\prime}$ will be denoted by $A_{n}^{\prime}, G_{n}^{\prime}$, and $H_{n}^{\prime}$, respectively.

The classical Ky Fan inequality reads as follows

$$
\frac{G_{n}}{G_{n}^{\prime}} \leqslant \frac{A_{n}}{A_{n}^{\prime}}
$$

(see, for example, [3]). A companion inequality to (1.1) has been obtained by Wang and Wang in [24], and it states that

$$
\frac{H_{n}}{H_{n}^{\prime}} \leqslant \frac{G_{n}}{G_{n}^{\prime}} .
$$

These two inequalities have stimulated the interest of several researchers, which resulted in many new results, refinements and improvements. The interested reader is referred to $[1,2,5,6,7,9,11,15,16,17,20,21,22]$.

Received 1st March, 2005

Copyright Clearance Centre, Inc. Serial-fee code: 0004-9727/05 \$A2.00+0.00. 
In the following by $w=\left(w_{1}, \ldots, w_{n}\right), w_{i} \geqslant 0(1 \leqslant i \leqslant n), w_{1}+\cdots+w_{n}=1$ denotes the $n$-tuple of weights. The weighted arithmetic, geometric, and harmonic means of $X \in \mathbb{R}_{+}^{n}$ with the weights $w$, denoted by $A(X, w), G(X, w)$ and $H(X, w)$, are defined in the usual way:

$$
\begin{aligned}
& A(X, w)=\sum_{i=1}^{n} w_{i} x_{i}, \quad G(X, w)=\prod_{i=1}^{n} x_{i}^{w_{i}} \\
& H(X, w)=\frac{1}{\sum_{i=1}^{n} w_{i}\left(1 / x_{i}\right)}
\end{aligned}
$$

Other weighted means used in this paper include the logarithmic mean $L(X, w)$ and the identric mean $I(X, w)$. Both means admit integral representations which are given below. Let

$$
E_{n-1}=\left\{\left(u_{1}, \ldots, u_{n-1}\right): u_{i} \geqslant 0(1 \leqslant i \leqslant n-1), u_{i}+\cdots+u_{n-1} \leqslant 1\right\}
$$

denote the Euclidean simplex, and for $\left(u_{1}, \ldots, u_{n-1}\right) \in E_{n-1}$, put

$$
u_{n}:=1-\left(u_{1}+\cdots+u_{n-1}\right) \text {. }
$$

Throughout the paper $n \geqslant 2$ and $\mu$ is an arbitrary probability measure on $E_{n-1}$. The natural weights $w_{i}(1 \leqslant i \leqslant n)$ of the measure $\mu$ are defined by

$$
w_{i}=\int_{E_{n-1}} u_{i} d \mu(u),
$$

where $d \mu(u)=\mu(u) d u_{1} \cdots d u_{n-1}$ and $u=\left(u_{1}, \ldots, u_{n}\right)$ (see, for example, [4]). The weighted logarithmic mean of $X$ is defined as

$$
L(X, w)=\left[\int_{E_{n-1}}(u \cdot X)^{-1} d \mu(u)\right]^{-i}
$$

where $u \cdot X=u_{1} x_{1}+\cdots+u_{n} x_{n}$ is the dot product of $u$ and $X$ (see, for example, [18]). The weighted identric mean is given by

$$
I(X, w)=\exp \left[\int_{E_{n-1}} \ln (u \cdot x) d \mu(u)\right]
$$

(see [18]).

An important probability measure on $E_{n-1}$ is the Dirichlet measure $\mu_{b}(u)$, where $b \in \mathbb{R}_{+}^{n}$. It is defined by

$$
\mu_{b}(u)=\frac{1}{B(b)} \prod_{i=1}^{n} u_{i}^{b_{i}-1}
$$


where $B(\cdot)$ stands for the multivariate beta function (see [4]). It is known that the natural weights $w_{i}$ of $\mu_{b}$ are given by

$$
w_{i}=b_{i} / c
$$

$(1 \leqslant i \leqslant n)$ where $c=b_{1}+\cdots+b_{n}$ (see $\left.[4]\right)$.

The weighted geometric mean also admits an integral representation

$$
G(X, w)=\left[\int_{E_{n-1}}(u \cdot X)^{-c} d \mu_{b}(u)\right]^{-1 / c}
$$

This follows from $[4,(6.6-6)]$. It is known that the weighted means mentioned above satisfy the inequalities

$$
\begin{aligned}
H(X, w) \leqslant G(X, w) & \leqslant L(X, w) \leqslant I(X, w) \\
& \leqslant A(X, w) \leqslant M_{p}(X, w)
\end{aligned}
$$

$(p \geqslant 1)$ where

$$
M_{p}(X, w)= \begin{cases}\left(\sum_{i=1}^{n} w_{i} x_{i}^{p}\right)^{1 / p}, & p \neq 0 \\ G(X, w), & p=0\end{cases}
$$

is the weighted power mean of order $p$ of $X \in \mathbb{R}_{+}^{n}$. Some of the means which appear in (1.10) satisfy the Ky Fan type inequalities. For instance, Sándor and Trif [22] have proven that

$$
\frac{G(X, w)}{G^{\prime}(X, w)} \leqslant \frac{I(X, w)}{I^{\prime}(X, w)} \leqslant \frac{A(X, w)}{A^{\prime}(X, w)} .
$$

(See also [21] for a special case of (1.12)).

Let the letters $G, L$, and $A$ stand for the unweighted geometric, logarithmic, and arithmetic means, respectively, of two positive numbers $x$ and $y$. Recall that

$$
L \equiv L(x, y)= \begin{cases}\frac{x-y}{\ln (x / y)}, & x \neq y \\ x, & x=y .\end{cases}
$$

In [16] the authors have proven that

$$
\frac{G}{G^{\prime}} \leqslant \frac{L}{L^{\prime}} \leqslant \frac{A}{A^{\prime}}
$$

$(x, y \leqslant 1 / 2)$. In addition to these inequalities, results involving differences of the reciprocals of means have been obtained. The following result

$$
\begin{aligned}
\frac{1}{H^{\prime}(X, w)}-\frac{1}{H(X, w)} & \leqslant \frac{1}{L^{\prime}(X, w)}-\frac{1}{L(X, w)} \\
& \leqslant \frac{1}{A^{\prime}(X, w)}-\frac{1}{A(X, w)}
\end{aligned}
$$


is established in [15]. The middle term in (1.14) provides a refinement of an earlier result of Sándor [20]. Alzer [2] has shown that

$$
\frac{1}{H^{\prime}(X, w)}-\frac{1}{H(X, w)} \leqslant \frac{1}{G^{\prime}(X, w)}-\frac{1}{G(X, w)} .
$$

The same author (see [1]) also proved that

$$
\frac{1}{H_{n}^{\prime}}-\frac{1}{H_{n}} \leqslant \frac{1}{G_{n}^{\prime}}-\frac{1}{G_{n}} \leqslant \frac{1}{A_{n}^{\prime}}-\frac{1}{A_{n}} .
$$

The goal of this paper is to obtain Ky Fan type inequalities for the means mentioned in this section and also for other means discussed in Section 5 . In Section 2, we give some lemmas which will be used throughout the sequel. Therein we introduce the so-called Ky Fan rules which, among other things, allow us to obtain inequalities for the differences of reciprocals of means. For instance, an application of Lemma 2.1, part (i) to (1.1) and (1.2) gives immediately Alzer's result (1.16). New Ky Fan type inequalities for the multivariate means are obtained in Section 3. Two refinements of the inequality (1.1) are presented in Section 4. The next section is devoted to the study of the Ky Fan type inequalities for certain means derived from $A_{n}, G_{n}$ and $H_{n}$. Section 6 deals with the Ky Fan type inequalities for the unweighted means of two variables.

\section{LEMMAS}

The lemmas presented in this section will be used in the remaining parts of this paper. We shall refer to the inequalities (2.1)-(2.3) of Lemma 2.1 as the Ky Fan rules. Our first result reads as follows.

LEMMA 2.1. Let $a, b, a^{\prime}$ and $b^{\prime}$ be positive numbers.

(i) If $a \leqslant b$ and $a / a^{\prime} \leqslant b / b^{\prime} \leqslant 1$ or if $b \leqslant a$ and $1 \leqslant a / a^{\prime} \leqslant b / b^{\prime}$, then

$$
\frac{1}{a^{\prime}}-\frac{1}{a} \leqslant \frac{1}{b^{\prime}}-\frac{1}{b}
$$

(ii) If $a^{\prime} \leqslant b^{\prime}$ and $a / a^{\prime} \leqslant b / b^{\prime}$, then

$$
a a^{\prime} \leqslant b b^{\prime}
$$

(iii) If $a / a^{\prime} \leqslant b / b^{\prime}$, then

$$
\frac{a}{a^{\prime}} \leqslant \frac{a+b}{a^{\prime}+b^{\prime}} \leqslant \frac{b}{b^{\prime}}
$$


and

$$
\frac{a}{a^{\prime}} \leqslant \frac{\sqrt{a b}}{\sqrt{a^{\prime} b^{\prime}}} \leqslant \frac{b}{b^{\prime}}
$$

Proof: For the proof of $(2.1)$ consider the case when $a \leqslant b$ and $a / a^{\prime} \leqslant b / b^{\prime} \leqslant 1$. It follows that $1 / b \leqslant 1 / a$ and also that $1-b / b^{\prime} \leqslant 1-a / a^{\prime}$. Multiplying corresponding sides of the last two inequalities gives the desired result. The remaining case can be established in the same way. For the proof of (2.2) we use the assumptions to obtain $a^{\prime} / b^{\prime} \leqslant b^{\prime} / a^{\prime}$ and $b^{\prime} / a^{\prime} \leqslant b / a$. Combining the last two inequalities we obtain $a^{\prime} / b^{\prime} \leqslant b / a$. Hence (2.2) follows. We shall prove now the inequalities (2.3). We have $a b^{\prime} \leqslant a^{\prime} b$. Adding $a a^{\prime}$ to both sides we obtain $a\left(a^{\prime}+b^{\prime}\right) \leqslant a^{\prime}(a+b)$. Hence the first inequality in $(2.3)$ follows. The second one is obtained from $a b^{\prime} \leqslant a^{\prime} b$ after adding to both sides $b b^{\prime}$. The first inequality in (2.4) is derived from $a / a^{\prime} \leqslant b / b^{\prime}$. Multiplying both sides by $a / a^{\prime}$ and next extracting square roots of the resulting expressions gives the assertion. The second inequality in (2.4) is obtained from $a / a^{\prime} \leqslant b / b^{\prime}$ by multiplying both sides by $b / b^{\prime}$ and followed by the root extraction.

LEMma 2.2. Let $f:[0,1] \rightarrow \mathbb{R}$ be a convex (concave) function. Then the function $g:[0,1 / 2] \rightarrow \mathbb{R}$, given by $g(x)=f(x)+f(1-x)$ is a decreasing (increasing) function on its domain.

Proof: Assume that the numbers $x$ and $y$ are such that $0<x<y \leqslant 1 / 2$. Then $x<y \leqslant 1-y<1-x$, that is, both numbers $y$ and $1-y$ can be written as a convex combination of $x$ and $1-x$, that is, $y=\alpha x+\beta(1-x)$ and $1-y=\beta x+\alpha(1-x)$ $(\alpha, \beta \geqslant 0, \alpha+\beta=1)$. Making use of the definition of the function $g$ together with the assumption of convexity of $f$ we obtain

$$
\begin{aligned}
g(y) & =f(y)+f(1-y) \leqslant \alpha f(x)+\beta f(1-x)+\beta f(x)+\alpha f(1-x) \\
& =f(x)+f(1-x)=g(x) .
\end{aligned}
$$

This completes the proof.

Lemma 2.3. Let $0<x<y \leqslant 1 / 2$ and let $x^{\prime}=1-x$ and $y^{\prime}=1-y$. If $p=c \ln (x / y)$ and $q=c \ln \left(x^{\prime} / y^{\prime}\right)$, where $c>0$, then the following inequalities

$$
\begin{aligned}
\frac{\sinh q}{q}<\frac{\sinh p}{p} & \\
\cosh q & <\cosh p
\end{aligned}
$$

and

$$
\frac{\tanh p}{p}<\frac{\tanh q}{q}
$$

are valid. 
Proof: We shall show first that the numbers $p$ and $q$ satisfy the inequalities

$$
-p>q>0
$$

We have $0<x<y \leqslant y^{\prime}<x^{\prime}<1$. This in turn implies that the logarithmic means of $(x, y)$ and $\left(x^{\prime}, y^{\prime}\right)$ satisfy the inequality $L(x, y)<L\left(x^{\prime}, y^{\prime}\right)$. Thus

$$
\frac{y-x}{\ln (y / x)}<\frac{x^{\prime}-y^{\prime}}{\ln \left(x^{\prime} / y^{\prime}\right)}=\frac{y-x}{\ln \left(x^{\prime} / y^{\prime}\right)} \text {. }
$$

Since $y-x>0, \ln (y / x)>\ln \left(x^{\prime} / y^{\prime}\right)$. Multiplying both sides by $c>0$ we obtain (2.8). To complete the proof of inequalities (2.5)-(2.7) we utilise the fact that the functions $\sinh t / t, \cosh t$ and $\tanh t / t$ are even functions on $\mathbb{R}$ and also that the first two are strictly increasing on $\mathbb{R}_{+}$while the third one is strictly decreasing on the stated domain. Thus we have

$$
\frac{\sinh (-p)}{-p}=\frac{\sinh p}{p}>\frac{\sinh q}{q}
$$

$\cosh (-p)=\cosh p>\cosh q$ and

$$
\frac{\tanh (-p)}{-p}=\frac{\tanh p}{p}<\frac{\tanh q}{q}
$$

The proof is complete.

We shall also need the following.

LEMMA 2.4. Let $f$ be a continuous function on the interval $[a, b]$ and assume that all derivatives $f^{(\ell)}(m)(\ell=1,2, \ldots)$ exist at $m=(a+b) / 2$. Then

$$
\frac{1}{b-a} \int_{a}^{b} f(t) d t=\frac{1}{2}[f(a)+f(b)]-\sum_{k=1}^{\infty} \frac{2 k}{(2 k+1) !}\left(\frac{b-a}{2}\right)^{2 k} f^{(2 k)}(m) .
$$

PROOF: Integrating Taylor's expansion for the function $f$ about the point $t=m$

$$
f(t)=f(m)+\sum_{k=1}^{\infty} \frac{(t-m)^{k}}{k !} f^{(k)}(m)
$$

we obtain

$$
\frac{1}{b-a} \int_{a}^{b} f(t) d t=f(m)+\sum_{k=1}^{\infty} \frac{1}{(2 k+1) !}\left(\frac{b-a}{2}\right)^{2 k} f^{(2 k)}(m) .
$$

Next we substitute $t=a$ and $t=b$ into (2.10) and add up the resulting expressions to obtain

$$
\frac{1}{2}[f(a)+f(b)]=f(m)+\sum_{k=1}^{\infty} \frac{1}{(2 k) !}\left(\frac{b-a}{2}\right)^{2 k} f^{(2 k)}(m) .
$$

Eliminating $f(m)$ between (2.11) and (2.12) gives the desired result (2.9). 


\section{Inequalities Involving Means of Several Variables}

In this section we give several Ky Fan type inequalities for means of several variables. For the sake of notation we shall write $A$ for the weighted arithmetic mean $A(X, w), L$ for the weighted logarithmic mean $L(X, w)$, et cetera. Recall that $X=\left(x_{1}, \ldots, x_{n}\right) \in \mathbb{R}_{+}^{n}$ where $x_{i} \leqslant 1 / 2,1 \leqslant i \leqslant n$.

Our first result reads as follows.

TheOREM 3.1. Let $f:[0,1] \rightarrow \mathbb{R}$ be a convex function. Then

$$
\begin{aligned}
f(H)+f(1-H) & \geqslant f(G)+f(1-G) \geqslant f(L)+f(1-L) \\
& \geqslant f(I)+f(1-I) \geqslant f(A)+f\left(A^{\prime}\right) .
\end{aligned}
$$

Inequalities (3.1) are reversed if $f$ is a concave function on $[0,1]$. If $F:(0,1] \rightarrow \mathbb{R}_{+}$is a log-convex function, then

$$
\begin{aligned}
F(H) F(1-H) & \geqslant F(G) F(1-G) \geqslant F(L) F(1-L) \\
& \geqslant F(I) F(1-I) \geqslant F(A) F\left(A^{\prime}\right) .
\end{aligned}
$$

Inequalities (3.2) are reversed if $F$ is a log-concave function and they become equalities if $F(x)=(1-x) / x$.

Proof: For the proof of the inequalities (3.1) we apply Lemma 2.2 to the five first members of (1.10) together with an obvious identity $A+A^{\prime}=1$. Inequalities (3.2) follow from (3.1) by letting $f(x)=\ln F(x)$. The last statement of the theorem is obvious.

COROLLARY 3.2. The following inequalities

$$
\begin{aligned}
\frac{1}{H}+\frac{1}{1-H} \geqslant \frac{1}{G}+\frac{1}{1-G} \geqslant \frac{1}{L}+\frac{1}{1-L} \geqslant \frac{1}{I}+\frac{1}{1-I} \geqslant \frac{1}{A}+\frac{1}{A^{\prime}}, \\
\frac{1}{G}+\frac{1}{G^{\prime}} \geqslant \frac{1}{L}+\frac{1}{1-L} \geqslant \frac{1}{I}+\frac{1}{1-I} \geqslant \frac{1}{A}+\frac{1}{A^{\prime}}, \\
\frac{1}{H}+\frac{1}{H^{\prime}} \geqslant \frac{1}{G}+\frac{1}{1-G} \geqslant \frac{1}{L}+\frac{1}{1-L} \geqslant \frac{1}{I}+\frac{1}{1-I} \geqslant \frac{1}{A}+\frac{1}{A^{\prime}},
\end{aligned}
$$

are valid.

Proof: Inequalities (3.3) follow immediately from (3.1) by letting $f(x)=1 / x$. Since $G+G^{\prime} \leqslant A+A^{\prime}=1, G^{\prime} \leqslant 1-G$ and $1 / G+1 /(1-G) \leqslant 1 / G+1 / G^{\prime}$. This in conjunction with (3.3) gives the chain of inequalities (3.4). Using the same argument as above, we have $H^{\prime} \leqslant 1-H$. Hence $1 / H+1 / H^{\prime} \geqslant 1 / H+1 /(1-H)$. Combining this with (3.3) yields (3.5).

A multiplicative version (with refinements) of the Ky Fan inequality is contained in the following. 
COROLLARY 3.3. We have

$$
\begin{aligned}
H(1-H) & \leqslant G(1-G) \leqslant L(1-L) \leqslant I(1-I) \leqslant A A^{\prime}, \\
G G^{\prime} & \leqslant L(1-L) \leqslant I(1-I) \leqslant A A^{\prime},
\end{aligned}
$$

and

$$
\begin{aligned}
\Gamma(H) \Gamma(1-H) & \geqslant \Gamma(G) \Gamma(1-G) \geqslant \Gamma(L) \Gamma(1-L) \\
& \geqslant \Gamma(I) \Gamma(1-I) \geqslant \Gamma(A) \Gamma\left(A^{\prime}\right),
\end{aligned}
$$

where $\Gamma$ stands for Euler's gamma function.

Proof: Inequalities (3.6) follow from (3.1) by letting $f(t)=\ln t$. Taking into account that $G^{\prime} \leqslant 1-G$ we obtain (3.7) from (3.6). It is well-known that the gamma function $\Gamma(x)$ is log-convex for $x>0$ (see, for example, [4]). Letting $F=\Gamma$ in (3.2) gives the inequalities (3.8).

In [19], Sándor has proven that the function $f(x)=[\Gamma(x)]^{1 / x}$ is convex for $x>0$. This in conjunction with (3.1) gives more Ky Fan type inequalities. We omit further details.

Before we shall state the next result let us introduce more notation. Also, we shall use the concept of an integral average of a function. For $Y=\left(y_{1}, \ldots, y_{n}\right) \in \mathbb{R}^{n}$, let $C=[\min (Y), \max (Y)]$ with $\min (Y)<\max (Y)$. Further, let $f: C \rightarrow \mathbb{R}$ be a continuous function and let $\mu$ be a probability measure on the Euclidean simplex $E_{n-1}$. We define

$$
F(\mu ; Y)=\int_{E_{n-1}} f(u \cdot Y) d \mu(u)
$$

Application of the mean-value theorem for integrals gives $F(\mu ; Y)=f(\xi \cdot Y)$, where $\xi=\left(\xi_{1}, \ldots, \xi_{n-1}, 1-\xi_{1}-\cdots-\xi_{n-1}\right)$ with $\left(\xi_{1}, \ldots, \xi_{n-1}\right) \in E_{n-1}$. Thus $F(\mu ; Y)$ can be regarded as the average value of the function $f$. When $\mu=\mu_{b}$, the average $F\left(\mu_{b}, Y\right)$ $\equiv F(b ; Y)$ is studied in detail in Carlson's monograph [4] and is called the Dirichlet average of the function $f$.

Also, recall that a function $f$ is said to be $n$-convex on $C$ if $f\left[z_{0}, \ldots, z_{n}\right] \geqslant 0$ for all choices of $n+1$ points $z_{0}, \ldots, z_{n}$ in $C$. Here $f\left[z_{0}, \ldots, z_{n}\right]$ stands for the $n$-th order divided difference of $f$.

The following refinement of Levinson's inequality (see [11]) appears in [14, Theorem 3.4]. Let $f:(0,1] \rightarrow \mathbb{R}$ be a 3-convex function. Then

$$
f\left(A^{\prime}\right)-f(A) \leqslant F\left(\mu ; X^{\prime}\right)-F(\mu ; X) \leqslant \sum_{i=1}^{n} w_{i}\left[f\left(x_{i}^{\prime}\right)-f\left(x_{i}\right)\right] .
$$

Inequalities (3.9) are reversed if $f$ is a 3 -concave function on $(0,1]$. 
The integral average of the power function $f(t)=t^{p}(t>0, p \in \mathbb{R})$ will be denoted by $R_{p}\left(\mu_{;} \cdot\right)$ and when $\mu=\mu_{b}$, the Dirichlet measure on $E_{n-1}$, we shall simply write $R_{p}\left(b_{i} \cdot\right)$ instead of $R_{p}\left(\mu_{b} ; \cdot\right)$. The latter average is also called the $R$-hypergeometric function. Its importance in the theory of special functions is well documented in Carlson's monograph [4]. If $\alpha>0$ and if $\alpha^{\prime}:=b_{1}+\cdots+b_{n}-\alpha>0$, then the $R$-hypergeometric function $R_{-\alpha}$ admits another integral representation (see $[4,(6.8-6)]$ )

$$
R_{-\alpha}(b ; X)=\frac{1}{B\left(\alpha, \alpha^{\prime}\right)} \int_{0}^{\infty} t^{\alpha^{\prime}-1} \prod_{i=1}^{n}\left(t+x_{i}\right)^{-b_{i}} d t .
$$

We shall use inequalities (3.9) to obtain bounds for the symmetric elliptic integrals of the first and the second kind. They are denoted by $R_{F}$ and $R_{G}$, respectively, and defined as follows

$$
R_{F}(x, y, z)=\frac{1}{2} \int_{0}^{\infty}[(t+x)(t+y)(t+z)]^{-1 / 2} d t
$$

and

$$
R_{G}(x, y, z)=\frac{1}{4} \int_{0}^{\infty}[(t+x)(t+y)(t+z)]^{-1 / 2}\left(\frac{x}{t+x}+\frac{y}{t+y}+\frac{z}{t+z}\right) t d t
$$

(see [4]). We assume that $x, y, z$ are positive numbers and at most one of them is 0 .

We have the following.

Proposition 3.4. Let $\mu$ be an arbitrary probability measure on $E_{n-1}$ and let $X \in \mathbb{R}_{+}^{n}$ with $x_{i} \leqslant 1 / 2$ for $1 \leqslant i \leqslant n$. If $0<p<1$ or if $p>2$, then

$$
\left(A^{\prime}\right)^{p}-A^{p}<R_{p}\left(\mu ; X^{\prime}\right)-R_{p}(\mu ; X)<\left(M_{p}^{\prime}\right)^{p}-M_{p}^{p} .
$$

Inequalities in (3.10) are reversed if either $p<0$ or if $1<p<2$ and they become equalities if $p=0$ or $p=1$ or $p=2$.

Proof: Let $f(t)=t^{p}(t>0, p \in \mathbb{R})$. It is easy to see that $f$ is 3 -convex if $0<p<1$ or if $p>2$ and $f$ is 3 -concave if either $p<0$ or $1<p<2$. The inequalities (3.10) follow from (3.9). If $p=0$, then all three members in (3.10) are equal to zero and they are all equal to $A^{\prime}-A$ if either $p=1$ or $p=2$.

COROLlary 3.5. The symmetric elliptic integrals $R_{F}$ and $R_{G}$ satisfy the $K y$ Fan type inequalities

$$
\left(A^{\prime}\right)^{-1 / 2}-A^{-1 / 2}>R_{F}\left(X^{\prime}\right)-R_{F}(X)>\left(M_{-1 / 2}^{\prime}\right)^{-1 / 2}-M_{-1 / 2}^{-1 / 2}
$$

and

$$
\left(A^{\prime}\right)^{1 / 2}-A^{1 / 2}<R_{G}\left(X^{\prime}\right)-R_{G}(X)<\left(M_{1 / 2}^{\prime}\right)^{1 / 2}-M_{1 / 2}^{1 / 2},
$$

where $X=\left(x_{1}, x_{2}, x_{3}\right)$ with $0<x_{i} \leqslant 1 / 2,1 \leqslant i \leqslant 3$. 
PROOF: We use Proposition 3.4 with $\mu=\mu_{b}$ where $b=(1 / 2,1 / 2,1 / 2)$ and $p=-1 / 2$ and $p=1 / 2$, respectively, because $R_{F}(\cdot)=R_{-1 / 2}(b ; \cdot)$ and $R_{G}(\cdot)=R_{1 / 2}(b ; \cdot)$ (see [4]).

For more bounds for the symmetric elliptic integrals the interested reader is referred to $[\mathbf{1 3}]$.

We close this section with two Ky Fan type inequalities for the differences of reciprocals of certain multivariate means.

The following inequality

$$
\frac{L}{L^{\prime}} \leqslant \frac{I}{I^{\prime}}
$$

is established in [7]. This result was known to the authors of this paper prior to the publication of [7]. Application of Lemma 2.1, part (i) to the last inequality and the second inequality in (1.12) gives

$$
\frac{1}{L^{\prime}}-\frac{1}{L} \leqslant \frac{1}{I^{\prime}}-\frac{1}{I} \leqslant \frac{1}{A^{\prime}}-\frac{1}{A} .
$$

A refinement of the first inequality in (1.16) is provided by

$$
\frac{1}{H_{n}^{\prime}}-\frac{1}{H_{n}} \leqslant \frac{1}{J_{n}^{\prime}}-\frac{1}{J_{n}} \leqslant \frac{1}{G_{n}^{\prime}}-\frac{1}{G_{n}}
$$

where

$$
J_{n}:=\frac{I_{n}(Y)}{G_{n}(Y)} H_{n}
$$

with $Y=\left(y_{1}, \ldots, y_{n}\right), y_{i}=x_{1} \cdot \ldots \cdot x_{i-1} x_{i+1} \cdot \ldots \cdot x_{n}(1 \leqslant i \leqslant n)$. In [15] the authors have proven that $H_{n} \leqslant J_{n} \leqslant G_{n}$ and also that

$$
\frac{H_{n}}{H_{n}^{\prime}} \leqslant \frac{J_{n}}{J_{n}^{\prime}} \leqslant \frac{G_{n}}{G_{n}^{\prime}}
$$

Application of Lemma 2.1, part (i) to (3.15) gives immediately the inequalities (3.14).

\section{Two Refinements of the Ky Fan Inequality}

The goal of this section is to establish two refinements of the Ky Fan inequality (1.1).

Let $C$ be an interval in $\mathbb{R}$. Recall that a function $f: C \rightarrow \mathbb{R}$ is said to be Jensen's convex (or $J$-convex) on $C$ if Jensen's inequality

$$
f\left(\frac{1}{n} \sum_{i=1}^{n} y_{i}\right) \leqslant \frac{1}{n} \sum_{i=1}^{n} f\left(y_{i}\right)
$$

is satisfied for all $y_{i}(1 \leqslant i \leqslant n)$ in $C$.

We need the following. 
Proposition 4.1. Let $x_{i} \in C, 1 \leqslant i \leqslant n+1$ and let $f: C \rightarrow \mathbb{R}$ be a $J$-convex function. If $\bar{x}_{n}=\left(x_{n+1}+(n-1) A_{n+1}\right) / n$, then

$$
\begin{aligned}
2 f\left(A_{n+1}\right) & \leqslant f\left(A_{n}\right)+f\left(\bar{x}_{n}\right) \\
& \leqslant \frac{f\left(x_{1}\right)+\cdots+f\left(x_{n+1}\right)+(n-1) f\left(A_{n+1}\right)}{n} .
\end{aligned}
$$

Also, if $x_{n}^{*}=\left(A_{n+1}+(n-1) x_{n+1}\right) / n$, then

$$
\begin{aligned}
n f\left(A_{n+1}\right) & \leqslant(n-1) f\left(A_{n}\right)+f\left(x_{n}^{*}\right) \\
& \leqslant \frac{(n-1)\left[f\left(x_{1}\right)+\cdots+f\left(x_{n+1}\right)\right]+f\left(A_{n+1}\right)}{n} .
\end{aligned}
$$

Proof: For the proof of the inequalities (4.1), let us notice that $A_{n+1}=\left(A_{n}+\bar{x}_{n}\right) / 2$. Using Jensen's inequality three times we obtain

$$
\begin{aligned}
f\left(A_{n+1}\right) & =f\left(\frac{A_{n}+\bar{x}_{n}}{2}\right) \leqslant \frac{f\left(A_{n}\right)+f\left(\bar{x}_{n}\right)}{2} \\
& \leqslant \frac{1}{2}\left[\frac{f\left(x_{1}\right)+\cdots+f\left(x_{n}\right)}{n}+\frac{f\left(x_{n+1}\right)+(n-1) f\left(A_{n+1}\right)}{n}\right] .
\end{aligned}
$$

Hence the assertion follows. In the proof of (4.2) we shall utilise the following identity $A_{n+1}=\left[(n-1) A_{n}+x_{n}^{*}\right] / n$. Again, we appeal to Jensen's inequality to obtain

$$
\begin{aligned}
f\left(A_{n+1}\right) & =f\left(\frac{(n-1) A_{n}+x_{n}^{*}}{n}\right) \leqslant \frac{(n-1) f\left(A_{n}\right)+f\left(x_{n}^{*}\right)}{n} \\
& \leqslant \frac{1}{n}\left[(n-1) \frac{f\left(x_{1}\right)+\cdots+f\left(x_{n}\right)}{n}+\frac{f\left(A_{n+1}\right)+(n-1) f\left(x_{n+1}\right)}{n}\right] .
\end{aligned}
$$

The desired result now follows.

The main result of this section is contained in the following.

Theorem 4.2. Let $X \in \mathbb{R}_{+}^{n+1}$ with $x_{i} \leqslant 1 / 2$ for $1 \leqslant i \leqslant n+1$ and let the numbers $\bar{x}_{n}$ and $x_{n}^{*}$ have the same meaning as in Proposition 4.1. Then

$$
\left(\frac{A_{n+1}^{\prime}}{A_{n+1}}\right)^{2} \leqslant \frac{A_{n}^{\prime}}{A_{n}} \bar{x}_{n}^{\prime} \leqslant\left(\frac{G_{n+1}^{\prime}}{\bar{x}_{n+1}}\right)^{1+1 / n}\left(\frac{A_{n+1}^{\prime}}{A_{n+1}}\right)^{1-1 / n}
$$

where $\bar{x}_{n}^{\prime}=1-x_{n}$. Also,

$$
\frac{A_{n+1}^{\prime}}{A_{n+1}} \leqslant\left(\frac{A_{n}^{\prime}}{A_{n}}\right)^{1-1 / n}\left(\frac{x_{n}^{*^{\prime}}}{x_{n}^{*}}\right)^{1 / n} \leqslant\left(\frac{G_{n+1}^{\prime}}{G_{n+1}}\right)^{1-1 / n^{2}}\left(\frac{A_{n+1}^{\prime}}{A_{n+1}}\right)^{1 / n^{2}} .
$$

where $x_{n}^{*^{\prime}}=1-x_{n}^{*}$.

REMARK. It is easy to see, using the Ky Fan inequality (1.1) that the third members in (4.3) and (4.4) are bounded above by $\left(G_{n+1}^{\prime} / G_{n+1}\right)^{2}$ and by $G_{n+1}^{\prime} / G_{n+1}$, respectively. Thus the inequalities (4.3) and (4.4) provide refinements of (1.1).

Proof: We shall use Proposition 4.1 with $C=(0,1 / 2]$ and $f(x)=\ln (1-x) / x$. Easy computations show that (4.3) follows from (4.1) while (4.4) is a consequence of (4.2). We omit further details. 
COROLLARY 4.3. The following inequalities

$$
\frac{A_{n}^{\prime}}{A_{n}} \leqslant\left(\frac{G_{n+1}^{\prime}}{G_{n+1}}\right)^{1+1 / n}\left(\frac{A_{n+1}^{\prime}}{A_{n+1}}\right)^{1-1 / n}
$$

and

$$
\left(\frac{A_{n}^{\prime}}{A_{n}}\right)^{1-1 / n} \leqslant\left(\frac{G_{n+1}^{\prime}}{G_{n+1}}\right)^{1-1 / n^{2}}\left(\frac{A_{n+1}^{\prime}}{A_{n+1}}\right)^{1 / n^{2}}
$$

are valid.

PROOF: It follows from the definitions of the numbers $\bar{x}_{n}$ and $x_{n}^{*}$ that both are positive and not bigger than $1 / 2$. This in turn implies that $\bar{x}_{n}^{\prime} \geqslant \bar{x}_{n}$ and $x_{n}^{*^{\prime}} \geqslant x_{n}^{*}$. The inequalities (4.5) and (4.6) now follow from (4.3) and (4.4), respectively.

Before we state the last result of this section, let us introduce more notation. We define

$$
a_{n}=\frac{1}{A_{n}}-\frac{1}{A_{n}^{\prime}} \text { and } h_{n}=\frac{1}{H_{n}}-\frac{1}{H_{n}^{\prime}} .
$$

COROLlary 4.4. We have

$$
2 a_{n+1} \leqslant a_{n}+\widetilde{x}_{n} \leqslant \frac{n+1}{n} h_{n+1}+\frac{n-1}{n} a_{n+1},
$$

where $\widetilde{x}_{n}=1 / \bar{x}_{n}-1 / \bar{x}_{n}^{\prime}$.

Proof: Inequalities (4.7) follow easily from (4.1) when $C=(0,1 / 2]$ and $f(x)=1 / x-1 /(1-x)$.

In $[20]$ the author has proven that $a_{n+1} \leqslant h_{n+1}$ (see also (1.16)). This in conjunction with (4.7) gives

$$
a_{n+1} \leqslant\left(a_{n}+\widetilde{x}_{n}\right) / 2 \leqslant h_{n+1} .
$$

\section{The Ky Fan Inequalities for a Certain family of Means}

Let $X \in \mathbb{R}_{+}^{n}$. In [6] the authors have studied some unweighted means of $X$. They are denoted by $A_{n}^{+}, G_{n}^{+}$and $H_{n}^{+}$and defined by $A_{n}^{+}=A_{n}(X+1), G_{n}^{+}=G_{n}(X+1)$ and $H_{n}^{+}=H_{n}(X+1)$, where $X+1=\left(x_{1}+1, \ldots, x_{n}+1\right)$. In the abovementioned paper, the authors have proven that

$$
\frac{H_{n}}{H_{n}^{+}} \leqslant \frac{G_{n}}{G_{n}^{+}} \leqslant \frac{A_{n}}{A_{n}^{+}}
$$

Recently, Govedarica and Jovanović [9] have shown that

$$
\frac{1}{H_{n}^{+}}-\frac{1}{H_{n}} \leqslant \frac{1}{G_{n}^{+}}-\frac{1}{G_{n}} \leqslant \frac{1}{A_{n}^{+}}-\frac{1}{A_{n}} .
$$

It is worth mentioning that the inequalities (5.2) follow immediately from (5.1) and Lemma 2.1, part (i), because $A_{n} / A_{n}^{+} \leqslant 1$. 
Assume now that $x_{i}>1,1 \leqslant i \leqslant n$ and let us define the unweighted means

$$
A_{n}^{-}=A_{n}(X-1), \quad G_{n}^{-}=G_{n}(X-1), \quad H_{n}^{-}=H_{n}(X-1),
$$

where $X-1=\left(x_{1}-1, \ldots, x_{n}-1\right)$. The main result of this section reads as follows.

THEOREM 5.1. The following inequalities

$$
1 \leqslant \frac{A_{n}}{A_{n}^{-}} \leqslant \frac{G_{n}}{G_{n}^{-}} \leqslant \frac{H_{n}}{H_{n}^{-}}
$$

and

$$
\frac{1}{A_{n}^{-}}-\frac{1}{A_{n}} \leqslant \frac{1}{G_{n}^{-}}-\frac{1}{G_{n}} \leqslant \frac{1}{H_{n}^{-}}-\frac{1}{H_{n}}
$$

hold true.

Proof: The first inequality in (5.3) is an immediate consequence of the trival identity $A_{n}=A_{n}^{-}-1$. In order to prove the second inequality in (5.3) we use the following inequality ([10, Theorem 64], [12, 3.2.34, p. 208])

$$
\prod_{i=1}^{n}\left(1+y_{i}\right)^{1 / n} \geqslant 1+\prod_{i=1}^{n} y_{i}^{1 / n}
$$

with $y_{i}=x_{i}-1(1 \leqslant i \leqslant n)$ to obtain $G_{n} \geqslant 1+G_{n}^{-}$. This in conjunction with $G_{n} \leqslant A_{n}$ and $A_{n}^{-}=A_{n}-1$ gives

$$
\frac{G_{n}}{G_{n}^{-}} \geqslant \frac{G_{n}}{G_{n}-1} \geqslant \frac{A_{n}}{A_{n}-1}=\frac{A_{n}}{A_{n}^{-}} .
$$

The last inequality in (5.3) is a special case of the following one

$$
\frac{G_{n}(X)}{G_{n}(X-a)} \leqslant \frac{H_{n}(X)}{H_{n}(X-a)}
$$

where now $X$ is an $n$-tuple of positive numbers such that $\min (X)>a>0$. Inequality (5.5) is reversed if $a<0$. In order to establish (5.5) we define a function $f(x)=\prod_{i=1}^{n} x_{i}^{1 / n}$ $\left(x_{i}>0\right.$ for $\left.1 \leqslant i \leqslant n\right)$. It is known that $f(x)$ is a concave function on $\mathbb{R}_{+}^{n}$. Thus its gradient $\nabla f$ satisfies the inequality

$$
(\nabla f(X)-\nabla f(Y)) \cdot(X-Y) \leqslant 0
$$

$\left(X, Y \in \mathbb{R}_{+}^{n}\right)$. Since $\nabla f(X)=(1 / n) \prod_{i=1}^{n} x_{i}^{1 / n}\left(\left(1 / x_{1}\right), \ldots,\left(1 / x_{n}\right)\right)$, the last inequality can be written as

$$
\sum_{k=1}^{n}\left(\frac{G_{n}(X)}{x_{k}}-\frac{G_{n}(Y)}{y_{k}}\right)\left(x_{k}-y_{k}\right) \leqslant 0 .
$$

Letting $Y=X-a(a>0)$ we obtain the desired result.

We close this section with a remark that the first inequality in (5.1) follows from (5.5) by letting $a=-1$. 


\section{Inequalities InVolving Means of Two Variables}

This section deals with the Ky Fan inequalities for certain unweighted means of two variables $x>0$ and $y>0$. To this end we shall always assume that $x \neq y$. For the sake of notation we shall often omit symbols of the variables of a mean. Thus $A$ will stand for the unweighted arithmetic mean of $x$ and $y$, et cetera.

Recall that the identric I mean of order one of two variables is defined as

$$
I \equiv I(x, y)=\exp \left(-1+\frac{x \ln x-y \ln y}{x-y}\right) .
$$

The extended logarithmic mean $E_{t}$ of order $t \in \mathbb{R}$ is defined as follows

$$
E_{t} \equiv E_{t}(x, y)= \begin{cases}{\left[\frac{x^{t}-y^{t}}{t(x-y)}\right]^{1 /(t-1)}} & t \neq 0,1 \\ L(x, y), & t=0 \\ I(x, y), & t=1 .\end{cases}
$$

The latter mean is a special case of the two-parameter family of means $S_{p, q}(p, q \in \mathbb{R})$ introduced by Stolarsky in [23]

$$
S_{p, q} \equiv S_{p, q}(x, y)= \begin{cases}\left(\frac{q}{p} \frac{x^{p}-y^{p}}{x^{q}-y^{q}}\right)^{1 /(p-q)} & p q(p-q) \neq 0 \\ {\left[L\left(x^{p}, y^{p}\right)\right]^{1 / p},} & p \neq 0, q=0 \\ {\left[I\left(x^{p}, y^{p}\right)\right]^{1 / p},} & p=q \neq 0 \\ G(x, y), & p=q=0 .\end{cases}
$$

It is easy to see that

$$
E_{t}=S_{t, 1}
$$

Another two-parameter family of means, denoted by $G_{p, q}$, has been introduced by Gini in [8]. They are denoted by $G_{p, q}$ and defined as follows

$$
G_{p, q} \equiv G_{p, q}(x, y)= \begin{cases}\left(\frac{x^{p}+y^{p}}{x^{q}+y^{q}}\right)^{1 /(p-q)}, & p \neq q \\ {\left[J\left(x^{p}, y^{p}\right)\right]^{1 / p},} & p=q \neq 0 \\ G(x, y), & p=q=0,\end{cases}
$$

where

$$
J(x, y)=\exp \left(\frac{x \ln x+y \ln y}{x+y}\right) .
$$

Other means used in this section are denoted by $s_{k}$ and $\sigma_{k}(k=1,2, \ldots)$ and they are defined as follows

$$
s_{k} \equiv s_{k}(x, y)=G^{1 / k} E_{1 / k}^{1-1 / k}
$$


and

$$
\sigma_{k} \equiv \sigma_{k}(x, y)=G^{1 / k} M_{1 / k}^{1-1 / k}
$$

Easy computations show that

$$
s_{k}(x, y)=\frac{1}{k} \sum_{i=1}^{k}\left(x^{\alpha_{i}} y^{\beta_{i}}+x^{\beta_{i}} y^{\alpha_{i}}\right),
$$

where $\alpha_{i}=(2 i-1) / 2 k$ and $\beta_{i}=1-\alpha_{i}, 1 \leqslant i \leqslant k$. Also,

$$
\sigma_{1}=s_{1}=G, \quad \sigma_{2}=s_{2}=\left(G M_{1 / 2}\right)^{1 / 2}=\frac{1}{2}\left(x^{1 / 4} y^{3 / 4}+x^{3 / 4} y^{1 / 4}\right) .
$$

We shall also use the Heronian mean which will be denoted by $\mathrm{He}$ and is defined as

$$
H e \equiv H e(x, y)=\frac{x+(x y)^{1 / 2}+y}{3} .
$$

It is easy to verify that $H e=S_{3 / 2,1 / 2}$.

The following result will be used in the sequel.

PROPOSITION 6.1. Let $\lambda=(t / 2) \ln (x / y), t \in \mathbb{R}$. Then

$$
\frac{\sinh \lambda}{\lambda}=\frac{L E_{t}^{t-1}}{G^{t}}
$$

and

$$
\cosh \lambda=\left(\frac{M_{t}}{G}\right)^{t}
$$

In particular, if $t=1 / k(k=1,2, \ldots)$, then

$$
\frac{\sinh \lambda}{\lambda}=\frac{L}{s_{k}}
$$

and

$$
\cosh \lambda=\frac{M_{1 / k}}{\sigma_{k}} .
$$

Proof: Formulas (6.10) and (6.11) are valid when $t=0$. Let $t \neq 0$. We have

$$
\begin{aligned}
\frac{\sinh \lambda}{\lambda} & =\frac{e^{\lambda}-e^{-\lambda}}{2 \lambda}=\frac{(x / y)^{t / 2}-(y / x)^{t / 2}}{t \ln (x / y)}=\frac{(x y)^{t / 2}\left[(x / y)^{t / 2}-(y / x)^{t / 2}\right]}{t \ln (x / y)(x y)^{t / 2}} . \\
& =\frac{x^{t}-y^{t}}{t \ln (x / y)(x y)^{t / 2}}=\frac{x-y}{\ln (x / y)} \frac{x^{t}-y^{t}}{t(x-y)(x y)^{t / 2}}=\frac{L E_{t}^{t-1}}{G^{t}} .
\end{aligned}
$$

Similarly,

$$
\begin{aligned}
\cosh \lambda & =\frac{1}{2}\left(e^{\lambda}+e^{-\lambda}\right)=\frac{1}{2}\left[(x / y)^{t / 2}+(y / x)^{t / 2}\right] \\
& =\frac{(x y)^{t / 2}\left[(x / y)^{t / 2}+(y / x)^{t / 2}\right]}{2(x y)^{t / 2}}=\frac{\left(x^{t}+y^{t}\right) / 2}{(x y)^{t / 2}}=\left(\frac{M_{t}}{G}\right)^{t}
\end{aligned}
$$


For the proof of $(6.12)$ we let $t=1 / k$ in $(6.2)$ to obtain

$$
E_{1 / k}^{1 / k-1}=\frac{k}{\sum_{i=1}^{k} x^{(k-i) / k} y^{(i-1) / k}}
$$

Making use of (6.7) we obtain

$$
\frac{E_{1 / k}^{1 / k-1}}{G^{1 / k}}=\frac{1}{s_{k}} .
$$

This in conjunction with (6.10) gives the assertion (6.12). Formula (6.13) follows from (6.11) by letting $t=1 / k(k \in \mathbb{N})$. We have

$$
\begin{aligned}
\cosh \lambda & =\frac{\left(x^{1 / k}+y^{1 / k}\right) / 2}{(x y)^{1 / 2 k}}=\frac{\left(x^{1 / k}+y^{1 / k}\right)\left(x^{1 / k}+y^{1 / k}\right)^{k-1} / 2^{k}}{(x y)^{1 / 2 k}\left(x^{1 / k}+y^{1 / k}\right)^{k-1} / 2^{k-1}} \\
& =\frac{\left[\left(x^{1 / k}+y^{1 / k}\right) / 2\right]^{k}}{(x y)^{1 / 2 k}\left[\left(x^{1 / k}+y^{1 / k}\right) / 2\right]^{k-1}}=\frac{M_{1 / k}}{G^{1 / k} M_{1 / k}^{1-1 / k}}=\frac{M_{1 / k}}{\sigma_{k}}
\end{aligned}
$$

where in the last step we have used formula (6.6). The proof is complete.

To this end we shall always assume that $0<x, y \leqslant 1 / 2$. We are in a position to prove the following.

THEOREM 6.2. Let $k=1,2, \ldots$. Then the following inequalities

$$
\begin{gathered}
\frac{H}{H^{\prime}}<\frac{\sqrt{H G}}{\sqrt{H^{\prime} G^{\prime}}}<\frac{\sqrt{H L}}{\sqrt{H^{\prime} L^{\prime}}}<\frac{G}{G^{\prime}}<\frac{s_{2}}{s_{2}^{\prime}}, \\
\frac{s_{k}}{s_{k}^{\prime}}<\frac{L}{L^{\prime}}<\frac{M_{1 / 2}}{M_{1 / 2}^{\prime}}<\frac{H e}{H e^{\prime}}<\frac{M_{2 / 3}}{M_{2 / 3}^{\prime}}<\frac{I}{I^{\prime}}<\frac{A}{A^{\prime}}<\frac{M_{2}}{M_{2}^{\prime}}<\frac{J}{J^{\prime}},
\end{gathered}
$$

and

$$
\frac{G}{G^{\prime}} \leqslant \frac{\sigma_{k}}{\sigma_{k}^{\prime}}<\frac{M_{1 / k}}{M_{1 / k}^{\prime}}
$$

are valid with equality in (6.16) when $k=1$.

Proof: The first inequality in (6.14) follows from the Wang-Wang inequality (1.2) and Lemma 2.1, part (iii) while the second inequality in (6.14) follows from $G / G^{\prime}<L / L^{\prime}$ (see $[7,16]$ ). Multiplying both sides by $H / H^{\prime}$ and next extracting the square roots, we obtain the desired result. For the proof of the third inequality in (6.14) we apply the Ky Fan inequality for the Gini and Stolarsky means

$$
\frac{G_{p, q}}{G_{p, q}^{\prime}}<\frac{S_{p, q}}{S_{p, q}^{\prime}}
$$

$(p+q<0)$ (see [16]). Letting $p=-1, q=0$ and next utilising definitions of the Gini means and Stolarsky means we obtain $G_{-1,0}=H$ and $S_{-1,0}=G^{2} / L$. Substituting into 
(6.17) gives the assertion. The last inequality in (6.14) can be established as follows. We use $G / G^{\prime}<M_{1 / 2} / M_{1 / 2}^{\prime}$ (see, for example, $[5,16]$ ). The first inequality in (2.9) together with $s_{2}=\left(G M_{1 / 2}\right)^{1 / 2}$ implies

$$
\frac{G}{G^{\prime}}<\left(\frac{G M_{1 / 2}}{G^{\prime} M_{1 / 2}^{\prime}}\right)^{1 / 2}=\frac{s_{2}}{s_{2}^{\prime}} .
$$

Here we have used the identity $M_{1 / 2}=E_{1 / 2}$. We shall establish now the chain of the inequalities (6.15). The first one follows by application of Lemma 2.3, with $p=(1 / 2 k) \ln (x / y)$ and $q=(1 / 2 k) \ln \left(x^{\prime} / y^{\prime}\right)$ to $(6.12)$. For the proof of the second inequality in $(6.15)$ we combine $(6.12)$ with $(6.13)$ to obtain

$$
\frac{\tanh \lambda}{\lambda}=\frac{L \sigma_{k}}{M_{1 / k} s_{k}} \text {. }
$$

Using (2.7) with $p$ and $q$ as defined earlier, we obtain

$$
\frac{L \sigma_{k}}{L^{\prime} \sigma_{k}^{\prime}}<\frac{M_{1 / k} s_{k}}{M_{1 / k}^{\prime} s_{k}^{\prime}} \quad(k=1,2, \ldots)
$$

Letting above $k=2$ and taking into account that $\sigma_{2}=s_{2}$ (see (6.8)), we obtain the desired result. The third inequality in (6.15) can be proven as follows. Assume without a loss of generality that $x<y$ and next define $t^{2}=y / x$ and $t^{\prime 2}=(1-y) /(1-x)$. Clearly $t>t^{\prime}$. Also, let

$$
f(t)=\frac{M_{1 / 2}\left(t^{2}, 1\right)}{\operatorname{He}\left(t^{2}, 1\right)}
$$

An easy computation shows that $f(t)=\frac{3}{4}\left(1+\frac{t}{t^{2}+t+1}\right)$ and also that $f^{\prime}(t)=\frac{3}{4} \frac{1-t^{2}}{\left(t^{2}+t+1\right)^{2}}$. Thus the function $f(t)$ is strictly decreasing when $t^{2}>1$. This in turn implies that

$$
\frac{M_{1 / 2}\left(t^{2}, 1\right)}{H e\left(t^{2}, 1\right)}<\frac{M_{1 / 2}\left(t^{\prime 2}, 1\right)}{H e\left(t^{\prime 2}, 1\right)}
$$

Replacing $t^{2}$ and $t^{\prime 2}$ by their defining expressions, next multiplying the numerators on both sides by $x$ and the denominators by $1-x$ and utilising homogeneity and symmetry of both means in their variables, we obtain the desired result. For the proof of the fourth inequality in (6.15) we still assume that $x<y$ and we let $t^{3}=y / x$ and $t^{\prime 3}=(1-y) /(1-x)$. Again we have $t>t^{\prime}$. Also, let

$$
g(t)=\frac{H e\left(t^{3}, 1\right)}{M_{2 / 3}\left(t^{3}, 1\right)}
$$

Logarithmic differentiation gives

$$
\frac{g^{\prime}(t)}{g(t)}=-\frac{3}{2} \frac{t^{1 / 2}(t-1)\left(t^{1 / 2}-1\right)^{2}}{\left(t^{3}+t^{3 / 2}+1\right)\left(t^{2}+1\right)}<0 .
$$


Thus the positive function $g(t)$ is decreasing for $t>1$. We follow the lines introduced in the proof of the previous inequality to obtain the assertion. The fifth inequality in (6.15) is established in a similar manner. With $t$ and $t^{\prime}$ as in the proof of the last inequality and with

$$
k(t)=\left[\frac{M_{2 / 3}\left(t^{3}, 1\right)}{I\left(t^{3}, 1\right)}\right]^{2 / 3}
$$

we have

$$
\frac{k^{\prime}(t)}{k(t)}=\frac{2 t^{2}}{\left(t^{3}-1\right)^{2}} s(t)
$$

where

$$
s(t)=3 \ln t-\frac{(t+1)\left(t^{3}-1\right)}{t\left(t^{2}+1\right)} .
$$

Letting $u=t^{3}$ in Karamata's inequality [12, 3.616, p. 272]

$$
\frac{\ln u}{u-1} \leqslant \frac{1+u^{1 / 3}}{u+u^{1 / 3}}
$$

we obtain $s(t) \leqslant 0$ for $t>1$. Thus the function $k(t)$ is strictly decreasing. The fifth inequality in (6.15) now follows by using the same argument as in the proof of the last two previous inequalities. The sixth and seventh inequalities in (6.15) are known (see (1.12) and [5], respectively). To prove the last inequality in (6.15) we proceed as follows. Again assume that $x<y$ and put $t=y / x$ and $t^{\prime}=(1-y) /(1-x)$. Also, let

$$
f(t)=\frac{M_{2}(t, 1)}{J(t, 1)}
$$

where the Gini mean $J$ is defined in (6.4). We have

$$
f(t)=\left(\frac{t^{2}+1}{2}\right)^{1 / 2} \frac{1}{t^{t /(t+1)}} .
$$

Hence

$$
\frac{f^{\prime}(t)}{f(t)}=\frac{h(t)}{(t+1)^{2}\left(t^{2}+1\right)}
$$

where $h(t)=t^{2}-1-\left(t^{2}+1\right) \ln t$. In order to prove that the function $f(t)$ is strictly decreasing for $t>1$ we use

$$
\frac{t-1}{\ln t}=L(t, 1)<\frac{t+1}{2}<\frac{t^{2}+1}{t+1},
$$

where the second inequality follows from the following one $(t+1)^{2}<2\left(t^{2}+1\right)$. It follows from (6.18) that $h(t)<0$ for $t>1$. This in turn implies that $f(t)$ is strictly decreasing on the stated domain. We shall prove now the inequalities (6.16). It follows from (6.6) that

$$
\frac{\sigma_{k}}{G}=\left(\frac{M_{1 / k}}{G}\right)^{1-1 / k}
$$


On the other hand, letting $t=1 / k$ in (6.11) we obtain

$$
\frac{M_{1 / k}}{G}=(\cosh \lambda)^{k}
$$

Combining the last two formulas we obtain

$$
\frac{\sigma_{k}}{G}=(\cosh \lambda)^{k-1} \text {. }
$$

Next we use inequality (2.5) of Lemma 2.3 to obtain the desired result. The second inequality in (6.16) is also proven with the aid of Lemma 2.3 and formula (6.13). We omit further details. The proof is complete.

More Ky Fan type inequalities can be obtained immediately from (6.14)-(6.16) by using the Ky Fan Rules (i)-(iii) of Lemma 2.1. We omit further details.

Our next result reads as follows.

THEOREM 6.3. The following inequalities

$$
\left(\frac{M_{1 / 2} G^{3}}{M_{1 / 2}^{\prime} G^{3}}\right)^{1 / 2}<\frac{L H e}{L^{\prime} H e^{\prime}}<\left(\frac{M_{1 / 2}}{M_{1 / 2}^{\prime}}\right)^{1 / 2}\left(\frac{M_{3 / 2}}{M_{3 / 2}^{\prime}}\right)^{3 / 2}
$$

hold true.

Proof: We use (6.10) with $t=3 / 2$ and also utilise $E_{3 / 2}^{1 / 2}=H e / M_{1 / 2}^{1 / 2}($ see $(6.2))$ to obtain

$$
\frac{\sinh \lambda}{\lambda}=\frac{L H e}{\left(M_{1 / 2} G^{3}\right)^{1 / 2}},
$$

where now $\lambda=(3 / 4) \ln (x / y)$. Application of the inequality (2.5) with $p=(3 / 4) \ln (x / y)$ and $q=(3 / 4) \ln \left(x^{\prime} / y^{\prime}\right)$, completes the proof of the first inequality in (6.19). In order to establish the second one we combine (6.10) with (6.11) to obtain

$$
\frac{\tanh \lambda}{\lambda}=\frac{L E_{t}^{t-1}}{M_{t}^{t}}
$$

where now $\lambda=(t / 2) \ln (x / y)$. Making use of Lemma 2.3 we obtain

$$
\frac{L E_{t}^{t-1}}{M_{t}^{t}}<\frac{L^{\prime}\left(E_{t}^{\prime}\right)^{t-1}}{\left(M_{t}^{\prime}\right)^{t}}
$$

Hence

$$
\frac{L}{L^{\prime}}\left(\frac{E_{t}}{E_{t}^{t}}\right)^{t-1}<\left(\frac{M_{t}}{M_{t}^{\prime}}\right)^{t}
$$

Letting $t=3 / 2$ and using $E_{3 / 2}^{1 / 2}=H e / M_{1 / 2}^{1 / 2}$ we obtain the assertion.

Neuman and Sándor [15] have proven that

$$
\frac{1}{H^{\prime}}-\frac{1}{L^{\prime}}<\frac{1}{H}-\frac{1}{L}
$$

The following result provides an improvement of the last inequality. We have 
THEOREM 6.4. The following inequality

$$
A^{\prime}\left(\frac{1}{H^{\prime}}-\frac{1}{L^{\prime}}\right)<A\left(\frac{1}{H}-\frac{1}{L}\right)
$$

is valid.

Proof: We shall use the following series expansion

$$
A\left(\frac{1}{H}-\frac{1}{L}\right)=\sum_{k=1}^{\infty} \frac{2 k}{2 k+1}\left(\frac{x-y}{x+y}\right)^{2 k} .
$$

For its proof we employ (2.9) with $a=x, b=y$, and $f(t)=1 / t$. Utilising the formula

$$
\frac{1}{L}=\frac{1}{x-y} \int_{y}^{x} \frac{d t}{t}
$$

we obtain

$$
\frac{1}{L}=\frac{1}{H}-\frac{1}{A} \sum_{k=1}^{\infty} \frac{2 k}{2 k+1}\left(\frac{x-y}{x+y}\right)^{2 k} .
$$

Hence the formula (6.21) follows. For the proof of $(6.20)$ we let

$$
u=\frac{x-y}{x+y} \text { and } v=\frac{x^{\prime}-y^{\prime}}{x^{\prime}-y^{\prime}},
$$

where $x^{\prime}=1-x$ and $y^{\prime}=1-y$. One can easily verify that $|v| \leqslant|u|$. Making use of (6.21) we obtain

$$
A^{\prime}\left(\frac{1}{H^{\prime}}-\frac{1}{L^{\prime}}\right)=\sum_{k=1}^{\infty} \frac{2 k}{2 k+1} v^{2 k} \leqslant \sum_{k=1}^{\infty} \frac{2 k}{2 k+1} u^{2 k}=A\left(\frac{1}{H}-\frac{1}{L}\right) .
$$

The proof is complete.

\section{REFERENCES}

[1] H. Alzer, 'Inequalities for arithmetic, geometric and harmonic means', Bull. London Math. Soc. 22 (1990), 362-366.

[2] H. Alzer, 'The inequality of Ky Fan and related results', Acta Appl. Math. 38 (1995), 305-354.

[3] E.F. Beckenbach and R. Bellman, Inequalities (Springer-Verlag, New York, 1965).

[4] B.C. Carlson, Special functions of applied mathematics (Academic Press, New York, 1977).

[5] F. Chan, D. Goldberg and S. Gonek, 'On extensions of an inequality among means', Proc. Amer. Math. Soc. 42 (1974), 202-207.

[6] E. El-Neweihi and F. Proschan, 'Unified treatment of some inequalities among means', Proc. Amer. Math. Soc. 81 (1981), 388-390.

[7] I. Gavrea and T. Trif, 'On Ky Fan's inequality', Math. Inequal. Appl. 4 (2001), 223-230. 
[8] G. Gini, 'Di una formula comprensiva delle medie', Metron 13 (1938), 3-22.

[9] V. Govedarica and M. Jovanović, 'On the inequalities of Ky Fan, Wang-Wang and Alzer', J. Math. Anal. Appl. 270 (2002), 709-712.

10] G.H. Hardy, J.E. Littlewood and G. Pólya, Inequalities, (2nd edition) (Cambridge Univ. Press, London and New York, 1952).

[11] N. Levinson, 'Generalization of an inequality of Ky Fan', J. Math. Anal. Appl. 8 (1964), 133-134.

[12] D.S. Mitrinović, Analytic inequalities (Springer-Verlag, Berlin, 1970).

[13] E. Neuman, 'Bounds for symmetric elliptic integrals', J. Approx. Theory 122 (2003), 249-259.

[14] E. Neuman, C.E.M. Pearce, J. Pečarić and V. Šimić, 'The generalized Hadamard inequality, g-convexity and functional Stolarsky means', Bull. Austral. Math. Soc. 68 (2003), 303-316.

[15] E. Neuman and J. Sándor, 'On the Ky Fan inequality and related inequalities I', Math. Inequal. Appl. 5 (2002), 49-56.

[16] E. Neuman and J. Sándor, 'Inequalities involving Stolarsky and Gini means', Math. Pannon. 14 (2003), 29-44.

[17] E. Neuman and J. Sándor, 'On the Schwab-Borchardt mean', Math. Pannon. 14 (2003), 253-266.

[18] J. Pečarić and V. Šimić, 'Stolarsky-Tobey mean in $n$ variables', Math. Inequal. Appl. 2 (1999), 325-341.

[19] J. Sándor, 'Sur la fonction Gamma', Publ. Centre. Rrech. Math. Pures., Neuchâtel, Ser. I 21 (1989), 4-7.

[20] J. Sándor, 'On an inequality of Ky Fan', (Preprint 90-7, Babes-Bolyai Univ.), pp. 29-34.

[21] J. Sándor, 'On the identric and logarithmic means', Aequationes Math. 40 (1990), 261-270.

[22] J. Sándor and T. Trif, 'A new refinement of the Ky Fan inequality', Math. Inequal. Appl. 2 (1999), 529-533.

[23] K.B. Stolarsky, 'Generalizations of the logarithmic mean', Math. Mag. 48 (1975), 87-92.

[24] W.-L. Wang and P.-F. Wang, 'A class of inequalities for symmetric functions', (in Chinese), Acta Math. Sinica 27 (1984), 485-497.

Department of Mathematics

Southern Illinois University

Carbondale, IL 62901-4408

United States of America

e-mail: edneuman@math.siu.edu

urladdr:

http://www.math.siu.edu/neuman/personal.html
Department of Pure Mathematics

Babes-Bolyai University

Ro-3400 Cluj-Napoca

Romania

e-mail: jsandor@math.ubbcluj.ro 